\title{
EMPIRICALLY ORIENTED APPROACH TO SPECTRAL CLASSIFICATION
}

M. S. Vardya

Tata Institute of Fundamental Research, Bombay

\section{ABSTRACT}

It is stressed that the ultimate aim of spectral classification is stellar classification. MKK classification is briefly described and possible additional parameters or dimensions discussed. Considering various new developments, a new approach to classification which is highly computer dependent, is suggested.

\section{INTRODUCTION}

There is no better place for making a confession than the Vatican. Let me start my talk by confessing that I have never classified any stellar spectra. However, let me hasten to add that I feel rather lost when I read the title of a paper on a given star, whose name and/or $\mathrm{HD}$ or some other catalogue number is given but no spectral class. As a theoretician, I am interested not only in its broad spectral classification, but also whether it is a variable (what class?) or not, whether the lines of $\mathrm{Ti}, \mathrm{Tc}$, and rare earths are visible or not, whether it shows UV or IR excess, etc. This information tells me the sub- or sub-sub-class to which the star may belong in a multi-dimensional matrix. One usually starts his theoretical pursuit with a minimum of information, i.e. the temperature, the surface gravity and the chemical composition, but our understanding is better, the more refined the sub-classification is. Though we start with spectral classification, one should not forget that our ultimate aim is stellar classification.

Empirical spectral classification is essentially based on the overa11 appearance of the spectra and depends on: 
1. Presence or absence of lines of various elements;

2. Ratio(s) of line intensities or rather darkening on the plate;

3. Ratio of lines and continuum darkening;

4. Width of the line - sharp or broad;

5. Type of lines - shallow or deep;

6. Presence or absence of emission lines.

The amount of information contained in the spectrum of a star depends on the wavelength coverage and the effective spectral dispersion, among other things.

The most commonly used "MKK" spectral classification is restricted to $3920-4900 \AA$ with a dispersion of about $125 \AA \mathrm{mm}^{-1}$ at $\mathrm{H} \gamma$ and has three dimensions. The temperature class is fairly well defined with about 70 subclasses; the luminosity class, on the other hand, has only about ten subclasses; the third dimension of surface chemical composition is the most $i 11$ defined (WN, WC, C, $S$, etc.), except that one assumes that for most of the stars, the surface elemental composition is similar to that in the solar photosphere.

\section{PARAMETERS IN STELLAR CLASSIFICATION}

What are the other dimensions or parameters, with which one can classify a star, to be useful in a variety of different investigations? The following may be worth considering: (a) mass; (b) age; (c) rotation; (d) macro-turbulent velocity; (e) magnetic field; (f) duplicity; (g) variability; (h) circumstellar she11/disc; (i) polarization. Let us consider these briefly.

\subsection{Mass and Age}

It is known that on the main sequence, as one proceeds from 0 , $B$ to $M$ stars, one is going from higher mass stars to lower mass stars. However, things are not fully clear off the main sequence. There are no specific feature(s) that one has found to be sensitive to mass only.

A similar thing can be said about age. On the main sequence, stars are, on the average, older as we go from early to late-type stars. Wilson and Skumanich (1964) have found for the main sequence stars that the chromospheric activity, as measured by the H-K emission intensities, decreases with age. This is restricted to stars of spectral class F7 and cooler and requires high dispersion ( $10 \AA \mathrm{mm}^{-1}$ ) to be observed. 
The difficulty with mass and age off the main sequence arises mainly from our poor understanding of the hydrodynamical aspects of stellar evolution, along with the neglect of curvature effect in the outer layers, which play very important roles off the main sequence.

\subsection{Rotation and Macro-turbulent Velocity}

Rotation and macroturbulence make the lines broad and shallow. As the broadening is due to Doppler effect, it is a function of wavelength. However, separation of rotation and macroturbulence is difficult (c.f. Gray 1975; Smith 1976) and the effect of rotational broadening also depends on the projection (sin i) effect. Shell and Be stars are fast rotators as compared to $B$ stars, and the rotational velocities of $K$ and $M$ stars are rather small relative to hot stars. Rapid rotation may cause gravity darkening and give rise to differences in temperature and excitation (c.f. Heap 1976).

\subsection{Magnetic Field}

The presence of a magnetic field broadens a line and may split it into $\pi$ and $\sigma$ components. AIl Ap stars are supposed to have surface magnetic fields; as these stars are also supposed to be rapidly rotating, the magnetic field has been measured only in sharp lined stars seen pole on. The magnetic field has also been observed in a few Am, M and S stars. The measurement of circular polarization has yielded information on the large magnetic field in white dwarfs. In a11, only in about 100 stars or so has a magnetic field been detected so far.

\subsection{Duplicity}

A large fraction of stars are double. However, spectral changes are expected only in close pairs. These effects may be due to the searchlight effect, tidal effect or due to the Roche lobe, coupled with the mass transfer effect. A very limited amount of information is available at the moment, except in a few special cases.

\subsection{Variability}

There are more than twenty-six thousand known variable stars; the amount of variability is different in different wavelength regions, and variation at different wavelengths need not be in phase. A single spectrum normally does not provide information about the variability; one needs a series of spectra or photometric data, spread over the proper interval of time, to provide full information about variability. 


\subsection{Circumstellar Shell/Disc}

Certain spectral features do suggest the existence of circumstellar shells or discs and provide information about mass loss. Normally these features can be observed only on high dispersion spectra.

\subsection{Polarization}

The polarization and the position of the electric vector has been measured from about two thousand odd stars but, to the best of my knowledge, no serious attempt has been made to correlate intrinsic stellar polarization with spectral features.

As the information available on the above mentioned parameters is very limited, the accuracy with which stars can be classified using one or more of the above parameters is rather minimal. It is therefore, not surprising that these parameters have not found general use so far. These parameters, however, are of immense importance and should become more common in the future scheme of classification, as more data are accumulated.

\section{FUTURE TRENDS}

What are the future trends that one can envisage at this juncture in empirical spectral classification?

There are four major developments, which I think are going to play important roles in spectral, or should I say, stellar classification in the years to come:

1. Our visual spectral horizon has broadened to X-ray and ultraviolet wavelengths at one end and to infrared (near and far), microwave and radio region at the other. And the orbiting of a large number of satellites, operating at different wavelength regions, has made it possible to have simultaneous observations at different wavelength bands.

2. Computers are here to stay. More and more telescopes and auxiliary instruments have on line computers for observing and quick data analysis.

3. The photographic plate is being replaced more and more by solid state detectors. As a result, more and more observations are becoming available in digitized form, which can be directly fed to the computer for reduction and analysis. 
4. Great strides have been made in low light detectors and more advances are expected in the future. This will result in an enormous increase in the number of stellar spectra that will become available for a given subclass.

One may add here another development, which, hopefully, will come true within the next decade. It is the launching of the Space Telescope, which will have higher spatial resolution than presently available from groundbased telescopes, and will provide data for a very large number of stars.

These new developments indicate that the time has come to make more and more use of the computer for classification purposes. The approach can be classical (supervised learning) or ab initio (unsupervised learning (c.f. Fu 1976).

\subsection{Classical Approach (supervised learning)}

Classically, one classifies the spectrum of a star by comparing it with the spectra of standard stars. One can therefore, store, in digitized form, the spectra of standard stars. These spectra, tagged with known classification, form a training set in the language of pattern recognition. It is usually a good practice to have a test set, also of known classification, to provide an estimate of the performance. Then classifying the spectrum of a given star essentially boils down to its comparison with the standard spectra, using the techniques of pattern recognition, with due allowance for errors. Pattern recognition can be based on a point by point comparison or on a few preselected matching criteria. Note that the classification will be more reliable if it is developed using the largest training set available.

If the spectrum of the star to be classified is at a higher dispersion than the stored standard spectra, one can reduce the dispersion to that of the standard set, though not vice versa. This will help in reducing the scatter in the derived spectral type, which according to Morgan and Keenan (1973) is principally due to the large range in the characteristics of the spectrograms used.

Though I have not seen it clearly spelt out (this may be just my ignorance), the present spectral classification is based on the basic tenet that the standard stars of the system define the properties of the spectral classes at all wavelengths and at all dispersions. As more and more stars, including standard stars, are being observed at different wavelength regions and at higher dispersions, one is faced with certain problems in classification. 
Stars with similar visual spectra, however, need not always show the same spectral properties in the other parts of the electromagnetic spectrum and vice versa. For example, a star classified as B2V may be an X-ray source also, whereas the standard star of the same spectral type may not. In such cases, we may either: (a) ignore the properties, so far as classification is concerned, derived from wavelength regions other than the visual; (b) tag $p$ or some other symbol to the $X$-ray star to denote features different than in the standard star; (c) form a new sub- or sub-sub-class.

In a similar way, two stars may belong to the same spectral class, based on their spectra taken at a given dispersion. The stars may, however, differ in certain respects when examined at higher dispersion (c.f. Seitter 1976). For example, one of the stars may be an $0 e$, the other 0ef. In such cases also, we have options as above.

However, as the aim of spectral classification is really stellar classification, we should opt for the third option, i.e., form new sub- or sub-sub classes. These new sub-classes may lead us to higher dimension in spectral classification. This also implies that we need to look at the problem of classification afresh.

\subsection{Ab Initio Approach (unsupervised learning)}

This approach essentially amounts to discovering a property of a collection of objects, without knowing the property a priori. The spectra of a large number of stars, chosen at random, are the collection, and the problem is to group them in clusters, depending on the similarities of features. This involves an iterative procedure for selection of features. For each cluster, average values of the feature measurements form a standard set for classification. The weight assigned to each feature will also have to be evaluated for computing the discriminants, the values of which determine the class to which a given spectrum belongs. The more stars are in the total collection and in each cluster, the more precise will be the classification, and subclassification.

Stars in globular clusters are old Population II objects with chemical composition different than the solar photospheric composition. The increased spatial resolution that will become available with the launching of the Space Telescope, should enable one to obtain spectra of a large number of stars in each cluster. Comparison among stars of globular clusters with different ages as well as with solar composition stars may provide a more accurate footing to the age parameter and relative elemental abundances in spectral classification. 
One may add here that for visual spectral classification, one may find that lower dispersion spectra are preferable to higher dispersion ones, But higher dispersion implies more information content. And if needed, the computer can always convert or degrade a higher dispersion spectrum into a lower dispersion one. Therefore, one expects to gain more information and insight the higher is the dispersion of the collection on which the spectral classification is based. It is humanly impossible to take into account all the details that a high dispersion spectrum presents; but the computer has no such problem. Therefore, use of high dispersion spectra in digitized form over many wavelengths is the only hope to add higher dimensions to the spectral classification in a satisfactory way.

\section{PLAN OF ACTION}

Assuming that most spectra will be available in digitized form or can be converted to it, one should define several levels of standard effective dispersions, such that as one proceeds from lower to higher dispersions the accuracy of spectral classification increases. What these standard dispersions should be can be decided by a joint meeting of the interested Commissions of the I. A. U.

Let us assume, for illustration, that these standard dispersions are: (A) $5000 \AA \mathrm{mm}^{-1}$; (B) $1000 \AA \mathrm{mm}^{-1}$; (C) $500 \AA \mathrm{mm}^{-1}$; (D) $100 \AA \mathrm{mm}^{-1}$; (E) $50 \AA \mathrm{mm}^{-1}$; (F) $10 \AA \mathrm{mm}^{-1}$; (G) $5 \AA^{-1}$; (H) $1 \AA \mathrm{mm}^{-1}$. It is not always possible to have the spectra of a given star at the highest dispersion, nor at these standard values of the dispersion.

Let us consider the spectrum of a star at dispersion $X$, which is between $E$ and $F$. This implies that we can classify it only up to the standard level $\mathrm{E}$. The procedure should be to reduce it to Level $\mathrm{A}$ and then classify it. At this dispersion it may not be possible to classify it in better than a few groups, and let us say, it is assigned group $i, i . e . A_{i}$. Now coming to level $B$, each of the level $A_{i}$ may be further subdivided, and this star may belong to, say, $B\left[A_{i}\right]_{j}$. Continuing this way to level $E$, the star will be classified as $E\left[D\left[C\left[B\left[A_{i}\right]_{j}\right]_{k}\right]_{1}\right]_{m}$. Some of the levels may be degenerate in a given case. If all the stars for which spectra are available are classified in this way, one will have an enormous amount of homogeneous data at any level of classification. Of course, the number of stars at level A will be maximum, the number decreasing as we proceed to levels $B, C, D$, etc. Note that similar techniques have been used in classifying fingerprints (Pavlidis 1977), mass spectra of hairs (Arunachalam 1978) and determination of molecular formulas from low resolution mass spectra (c.f. Kowalski 1974), to name just a few applications. 
Development of such a computer code to classify stars may require a few man years or more, but is, as I see it, essential. And in developing such a code, one can try to take advantage of both qualitative and quantitative spectral classifications and minimize the disadvantages of the two procedures (Barry, Cromwell and Schoolman 1977).

Concluding, let me once again state, that the time is ripe to define the standard values of the dispersions to be used, use computers more and more to classify stars, and to tag each star with as many parameters as possible.

\section{ACKNOWLEDGEMENT}

It is a pleasure to thank Mr. J. Arunachalam and Dr. S. Ramani for very useful discussion on the computer aspect of classification, and to Dr. M. K. V. Bappu, Dr. K. S. Krishna Swamy, Dr. Janet R. Lesh and Dr. S. P. Tarafdar for their very valuable comments on an earlier draft of the manuscript.

\section{REFERENCES}

Arunachalam, J. (1978). Private communication.

Barry, D. C., Cromwe11, R.H. and Schoolman, S. (1977). Astrophys. J. 212, 462 .

Fu, K. S. (1976). in Digital Pattern Recognition, K. S. Fu, ed., Springer-Verlag, Ber1in, p. 1 .

Gray, D. F. (1975). Astrophys. J. 202, 148.

Heap, S. R. (1976). in IAU Symposium No. 70, Be and She11 Stars, A. Slettebak, ed., Reide1; Dordrecht, p. 165.

Kowalski, B. C. (1974). in Computers in Chemical and Biochemical Research, C. E. Klopfenstein and C. L. Wilkins, eds., Academic Press, New York, p. 1.

Morgan, W. W. and Keenan, P. C. (1973). A. Rev. Astr. Astrophys. 11, 29.

Pavlidis, T. (1977). Stmuctural Pattern Recognition, Springer-Verlag, Berlin.

Seitter, W. C. (1976). in IAU Symposium No. 72, Abundance Effects in Classification, B. Hauck and P. C. Keenan, eds., Reide1; Dordrecht, p. 129 .

Smith, M. A. (1976). Astrophys. J. 208, 487.

Wilson, 0. C. and Skumanich, A. (1964). Astrophys. J. 140, 1401. 


\section{DISCUSSION}

Garrison: It is essential to understand that the MK standards define the MK system in the MK domain as defined by Morgan and Keenan in their papers at this meeting. There is no assumption that they will be valid for all wavelength regions and all dispersions, though certainly they are likely to be among the better defined stars because they have better data in general. *

Vardya: Thanks, I stand corrected.

Coyne: You have referred to polarization as a means of understanding stellar atmospheres. In the first place we think of polarization as a probe of stellar magnetic fields. But the application is much broader. Measurements of intrinsic polarizations are becoming a valuable means of probing various atomic and molecular absorption processes with optical depth. I give two examples: (1) in Mira we have just recently (McLean, Coyne and Serkowski, 1978, Ap. J., in press) discovered that the HB emission flux is linearly polarized $6.8 \%$ which is four times the continuum polarization; (2) in $\mu$ Cep (McLean and Coyne, 1978, Ap. J. Letters, in press) we find that the linear polarization across two Tio bands at $\lambda<5500 \AA$ decreases and for three bands at $\lambda>5500 \AA$ it increases. In general the polarization produced by scattering from electrons, molecules and/or grains in modified by discrete absorption and emission. Thus we may have a means of discriminating the depth at which these processes take place. We need not pursue this further at this conference but you should be aware of the promising new field of stellar spectropolarimetry.

* See related comments by Janet Lesh and Nolan Walborn on page 346, the statement by Vardya on pages 331-2 and by Code on page 423, second paragraph. 